\title{
Diacronie
}

Studi di Storia Contemporanea

$N^{\circ} 15,3 \mid 2013$

Spazi, percorsi e memorie

\section{El Cuarto Gobierno de Fanfani y la apertura a la Izquierda}

Un acercamiento histórico

\section{Matteo Anastasi}

\section{OpenEdition}

\section{Journals}

\section{Edición electrónica}

URL: http://journals.openedition.org/diacronie/538

DOI: $10.4000 /$ diacronie. 538

ISSN: 2038-0925

\section{Editor}

Association culturelle Diacronie

Referencia electrónica

Matteo Anastasi, «El Cuarto Gobierno de Fanfani y la apertura a la Izquierda », Diacronie [En línea], N

15,3 | 2013, documento 11, Publicado el 01 octubre 2013, consultado el 10 diciembre 2020. URL:

http://journals.openedition.org/diacronie/538; DOI : https://doi.org/10.4000/diacronie.538 


\section{Diacronie}

\section{1/}

\section{El Cuarto Gobierno de Fanfani y la apertura a la Izquierda}

\section{Un acercamiento histórico}

Matteo ANASTASI*

En este ensayo se examina la situación política italiana durante el inicio de la década de los sesenta. Se trata de un momento de cambio profundo que culminará con la formación del cuarto gobierno Fanfani, ejecutivo que se abrirá a los socialistas, rompiendo la precedente tradición ligada al "centrismo", elemento dominante en el panorama político italiano desde el final de los años cuarenta. Objectivo de este estudio es recoger un balance del experimento de gobierno encabezado por el estadista aretino, luces y sombras de un ejecutivo que actuó bajo la mirada atenta de "derechas" e "izquierdas" contrarias a la alianza entre democristianos y socialistas.

\section{Introducción}

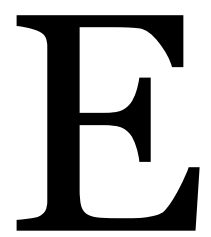

1 tema central, en torno al que se desarrolló toda la actividad política de la tercera legislatura italiana (1958-1963) fue la operación de apertura a la izquierda. La nueva mayoría, que nació del encuentro entre socialistas y católicos, se manifestó por primera vez en ocasión del voto de confianza al cuarto gobierno Fanfani, el 10 de marzo de 1962, cuatro años después de las elecciones políticas de 1958. Se llegó a la realización del primer gobierno de apertura a la izquierda después de muchas incertidumbres y un lento y difícil trabajo preparatorio, durante el cual «la actividad de los partidos, o mejor, de los grupos y de las corrientes en su seno, y la evolución natural de la situación política desempeñaron un papel igualmente 
determinante». En el seno, tanto de la Democracia Cristiana (DC), como del Partido Socialista Italiano (PSI), se produjeron tendencias muy fuertes contrarias a la apertura, más por su influencia que por su consistencia numérica. La misma situación se pudo verificar en ambos electorados; tanto el socialista como el democristiano observaron con expetación el acercamiento gradual entre los dos partidos. Los años de acción propagandista habían contribuido a presentar a los socialistas como sólidos aliados del Partido Comunista Italiano (PCI), y a la DC como principal defensor de los intereses del capital y de la Iglesia Católica.

\section{1. ¿Por qué una abertura hacia la izquierda?}

Uno de los principales temas, sobre el que se fundó el proyecto reformista del centro-izquierda, fue el de los desequilibrios que el rapidísimo desarrollo económico de los años precedentes había creado dentro del País. La exposición más notable de esta situación fue la Nota adicional de Ugo La Malfa, Ministro del Balance y de la Programación Económica del cuarto gobierno Fanfani y líder del Partido Republicano ${ }^{2}$, presentada en el Parlamento el 22 de mayo de 1962르 dos meses después del nacimiento del primer gobierno de apertura a la izquierda. Era la primera vez que en un texto oficial se exponía de forma directa e inderogable el problema de los desequilibrios creados por el modelo de desarrollo: «Quien tiene la responsabilidad en la política económica del País no puede ignorar que tal impetuoso desarrollo se ha visto acompañado de la persistencia de situaciones sectoriales, regionales y sociales de subdesarrollo y de retraso económico que, evidentemente, no consiguen obtener impulso suficiente de la expansión general del sistema»4. El modelo de desarrollo mantenido por el fuerte dinamismo de las empresas, la expandida capacidad de la industria en el mercado internacional y por la amplia disponibilidad de fuerza de trabajo - era el responsable, según la Nota adicional, de desequilibrios como la desvalorización del papel de la agricultura en la economía, el gran distanciamiento entre Norte y Sur, los movimientos incontrolables de personas que despoblaban

${ }^{1}$ MAMMARELLA, Giuseppe, L’Italia contemporanea 1943-1988, Bologna, Il Mulino, 2000, p. 255.

2 Partito Repubblicano Italiano (PRI) que constituía, junto con el Partito Socialista Democratico Italiano (PSDI) dirigido por Giuseppe Sagarat, uno de los dos grupos políticos que apoyaron a la DC dentro del ejecutivo encabezado por Amintore Fanfani durante el bienio 19611962.

3 Cfr. VOULGARIS, Yannis, L'Italia del centro-sinistra 1960-1968, Roma, Carocci, 1998, p. 98.

4 LA MALFA, Ugo, Problemi e prospettive dello sviluppo economico italiano. Nota aggiuntiva, in MINISTERO DEL BILANCIO E DELLA PROGRAMMAZIONE ECONOMICA, La programmazione economica in Italia, vol. 2, Roma, Istituto poligrafico dello Stato, 1967, p. 89. 
algunas regiones mientras que saturaban otras, y el insuficiente aumento de los servicios y consumos sociales respecto a los privados. Sin embargo, este audaz diagnóstico y ejercicio de autocrítica sobre la situación del País no constituyó una declaración pesimista; al contrario, el centro-izquierda en esos tiempos se afianzaba en un periodo de desarrollo que nadie hubiera previsto en los primeros años de la posguerra. Por otra parte, en 1962 el "milagro económico" se encontraba en pleno apogeo y existía un fuerte clima de confianza. De cualquier manera, como el mercado por sí sólo no podía promover la superación de las desigualdades, ni conferir unas características diferentes al desarrollo, esta labor correspondía al Estado y, en particular, al nuevo ejecutivo encabezado por Fanfani. Los medios de esa política debían ser la programación y el impulso coordinado de una serie de reformas. La intervención del Estado sobre la economía del País se hacía necesaria, afirmaba La Malfa, ya que «incluso aceptando que el desarrollo espontáneo acabará, antes o después, invirtiendo en las zonas y los sectores menos desarrollados del País, la resolución de los problemas hoy abiertos a nuestra economía se delinearía en un lapso de tiempo que sin duda debemos considerar muy dilatado para las exigencias y las expectativas legítimas de una parte muy considerable de la población italiana»5. La programación económica debía abarcar todos los sectores pero, en la Nota adicional, se señalaron algunas prioridades: la agricultura y el Sur, el refuerzo de los consumos sociales, con particular atención a la instrucción, la sanidad y la vivienda.

El programa del cuarto gobierno Fanfani contó con el apoyo parlamentario del PSI. Un respaldo que inauguró la era de la apertura a la izquierda y que se logró después de largas negociaciones que se sellaron con la aceptación, por parte del futuro ejecutivo, de algunas de las principales peticiones socialistas: instancias que, dos meses antes del discurso de La Malfa, en marzo de 1962, acabaron por integrarse en el programa político presentado por el gobierno en el Parlamento. Las reivindicaciones de los socialistas comprendían: «la nacionalización de la industria eléctrica, la adopción de una política económica de programación, la institución del ordenamiento regional según los artículos de la Constitución, medidas para mejorar el nivel de vida en las zonas rurales y a favor de la propiedad agrícola y un proyecto para la democratización de la escuela» 6 .

La nacionalización de la industria eléctrica era claramente un objetivo político. A pesar de que la operación se hubiera justificado con la necesidad de disminuir las tarifas para permitir un aumento del consumo y extender la red a zonas del Sur

\footnotetext{
5 Ibídem, p. 93.

${ }^{6}$ MAMMARELLA, Giuseppe, op. cit., p. 256.
} 
económicamente deprimidas, sus objetivos reales fueron otros. La nacionalización de las empresas productoras de energía eléctrica, que seguía a la constitución del monopolio de Estado del petróleo y el gas metano (ENI) y de un ente para la producción de energía atómica (CNEN), llevada a cabo por los gobiernos precedentes, formaba parte de una política a largo plazo para la gestión estatal de todas las fuentes de energía. En la implantación de esa política estaban particularmente interesados los socialistas y las corrientes de la izquierda católica, que la concebían como un punto de transición lógico hacia la programación económica. El control estatal de la producción y de la distribución de la energía eléctrica hubiera constituido un instrumento eficaz para influenciar las decisiones de la industria privada, orientándolas en la dirección preferida y coordinándolas con los objetivos del plan económico?

El segundo objetivo del PSI, fue la adopción de una política económica de programación, pretendía conseguir una más amplia participación de las clases trabajadoras en la vida política nacional, y estaba estrechamente vinculado a otros puntos del programa: la institución del ente "región" y la democratización de la escuela pública. La creación de las regiones habría articulado una notable descentralización de la vida política y administrativa; ésta hubiera favorecido a los partidos populares, mejor organizados a nivel local, y habría limitado la influencia de las grandes concentraciones de poder económico, por lo que resultaba más fácil ejercer presiones e influencias en la medida en que el poder de decisión se concentraba en pocos órganos centrales.

Un sistema escolar abierto a todas las clases sociales produciría efectos a más largo plazo. Este ofrecería una instrucción en todos los niveles educativos a los estudiantes meritorios y modificaría los viejos programas de estudio que reflejaban una concepción elitista de la cultura, favoreciendo a los estudiantes que provenían de las clases privilegiadas, educados en ambientes más $\operatorname{cultos}^{8}$.

Las medidas a favor de los campesinos, junto con las peticiones de un aumento en la cuantía de las pensiones para ciertas categorías profesionales, eran reivindicaciones que tenían el objetivo de asegurar beneficios inmediatos para las clases trabajadoras y una redistribución más justa de la renta nacional. Estos son los puntos fundamentales del programa de gobierno, objeto de largas negociaciones entre DC y PSI, que debían constituir el terreno de prueba para el primer experimento de colaboración entre los dos partidos.

\footnotetext{
7 Cfr. TAMBURRANO, Giuseppe, Storia e cronaca del centro-sinistra, Milano, Feltrinelli, 1971, pp. 182-202.

8 Cfr. POMBENI, Paolo, I partiti e la politica dal 1948 al 1963, in SABBATUCCI, Giovanni, VIDOTTO, Vittorio (a cura di), Storia d'Italia, vol. 5, La Repubblica: 1943-1963, Roma, Laterza, 2004, pp. 230-231.
} 
Además de la evidente necesidad de reformas para resolver las dificultades que estaba atravesando el País, otra motivación fundamental que originó el acuerdo entre democristianos y socialistas fue la evolución interna a estas dos formaciones políticas: la colaboración entre DC y PSI constituía, de hecho, «el desbloqueo natural de un largo proceso evolutivo que implicaba a ambos partidos» 9 . La convergencia se fundamentaba en la limitada legitimación del "centrismo", que desde la segunda posguerra había dominado la escena política italiana. Tal desgaste se hacía manifiesto con la existencia y, al mismo tiempo, el aislamiento de una fuerte oposición de izquierda. De hecho, gobernaba permanentemente una formación, la democristiana, que acumulaba en torno al 50\% del respaldo electoral y que no reconocía a las demás fuerzas políticas, ya estuvieran a su derecha o a su izquierda, el derecho a sustituirle. Por ello, era necesario invertir la tendencia: para los democristianos que sostenían que la apertura a la izquierda era un argumento sólido, significaba la necesidad de ampliar las bases sociales del Estado; para los socialistas, que representaban en ese momento el tercero partido de Italia tras el católico, era válida la consideración de que, gracias a la revisión puesta en marcha, antes o después los dos partidos obreros (PSI y PCI) se escindirían de la vieja formación de oposición, que carecía ya entonces de perspectivas ${ }^{10}$. Para ambos partidos, por tanto, la entrada de los socialistas en el gobierno era un objetivo consecuente a la respectiva evolución reformista.

La primera persona que planteó abiertamente el problema de la ampliación de las bases del Estado fue, en nombre del sector más a la izquierda de la DC, Giovanni Gronchi, que ya en 1954, poco antes de ser elegido presidente de la República, afirmó en un discurso oficial: «Es necesario para los intereses del País y responde a la naturaleza y a las misiones de la DC, afrontar el problema de una alternativa política, después de que la política centrista, de equilibrio, se demuestra superada por los hechos y por las necesidades que surgen [...] De la misma manera, le espera a la DC, como consecuencia de su posición de partido líder de la coalición, adaptar con diligencia la acción propia a la necesidad de atraer nuevas fuerzas a la órbita de la política democrática y del Estado republicano ${ }^{11}$.

Dentro de la izquierda católica se distinguían dos maneras diferentes de aproximarse al problema PSI, que también mostraban concepciones diferentes del carácter de la DC y de su relación con la Iglesia. Ambas aspiraban a la colaboración con el PSI con el objetivo de reforzar los grupos y las políticas reformistas. La primera de

9 VOULGARIS, Yannis, op. cit., p. 107.

${ }_{10}$ Cfr. Ibidem, p. 102.

11 TAMBURRANO, Giuseppe, op. cit., p. 8. 
las dos tendencias, de la que formaba parte Gronchi, era la expresión del elemento "populista” de la cultura católica; el carácter popular que debía tener la DC como partido católico, se habría materializado mediante la colaboración - necesaria, debido a la limitada aprobación alcanzada por el centrismo - con el PSI, como representante de parte de las masas populares de trabajadores. La otra tendencia, expresión sobre todo de la corriente democristiana conocida como Amici di Base, se posicionaba en una concepción más laica del carácter de la DC. La aproximación al PSI se produciría en el marco de una cultura democrática reformista, que habría reforzado las tendencias progresistas y anti-fundamentalistas de la DC.

El proceso de acercamiento entre democristianos y socialistas vivió un momento crucial en 1956, al producirse la primera ruptura seria de la alianza entre PCI y PSI, que hasta ese momento había sido bastante sólida. En ese año, el líder socialista Pietro Nenni comunicó al Parlamento que «un motivo profundo de desacuerdo [se había] producido entre el PSI y los compañeros comunistas»12 al condenar éste «sin ninguna reticencia» la represión soviética en Hungría, condena proclamada de forma diferencial por el secretario del PCI Palmiro Togliatti y del sector más a la izquierda del PSI ${ }^{13}$. El desacuerdo sobre los hechos de Hungría alimentó la separación entre el PSI y el PCI también en la política interior. Según los socialistas, la adhesión de los comunistas al modelo soviético dinamitaba las expectativas de la izquierda, debido a que éstas, en Italia, estaban supeditadas al juego democrático ${ }^{14}$. Durante el XXXII Congreso Nacional del PSI, que tuvo lugar en Venecia el 6 de febrero de 1957, Nenni se distanció definitivamente de los comunistas, proclamando la autonomía del PSI, y declarando que su acción aspiraba a una posibilidad de gobierno que se basara en un pacto con las fuerzas populares católicas, quienes tenían objetivos democráticos comunes: de esa manera, el líder socialista emprendía definitivamente el camino de acercamiento a la DC.

\section{Los adversarios al nuevo curso}

Los opositores naturales de la nueva trayectoria que se estaba delineando - la del centro-izquierda - eran las fuerzas de la derecha económica y, obviamente, los

${ }_{12}$ BAGET BOZZO, Gianni, Il partito cristiano e l'apertura a sinistra. La DC di Fanfani e di Moro, 1954-1962, Firenze, Vallecchi, 1977, p. 99.

${ }^{13}$ Se trataba de los llamados "carristas", exponentes de los sectores filo-soviéticas del PSI, llamados así porque habían justificado la intervención de los carros armados de la Armada Roja durante la revolución magiara.

${ }_{14}$ Cfr. PARTIDO SOCIALISTA ITALIANO, XXXII Congresso Nazionale, Milano, Edizioni «l'Avanti!», 1957, pp. 123-125. 
comunistas $^{15}$. Las primeras, veían en el programa de apertura a la izquierda una amenaza dirigida a posiciones de poder y de influencia contra las que, la nueva política de reformas, habría luchado, intentándolas limitar. La campaña en contra de la alianza política entre socialistas y católicos se produjo, «utilizando todos los medios» ${ }^{16}$, a través de la prensa, los grupos de presión y los representantes de los intereses económicos en los partidos conservadores y en el de mayoría relativa, quien manifestaba las serias dudas sobre las consecuencias de la nueva política presentes en amplios sectores empresariales.

Cuando la ofensiva de las fuerzas conservadoras se adentraba en la fase más intensa, le falló su apoyo más sólido: el del Vaticano, cuya influencia en el partido de mayoría había sido hasta entonces determinante. Después de la elección de Juan XXIII como Sumo Pontífice, que tuvo lugar el 28 de octubre de 1958, se observaron claras indicios de la profunda evolución que estaba produciéndose dentro de la Iglesia católica. Este avance, además de proclamar algunos de los principios fundamentales de la doctrina, que el Concilio de 1962 se preocupó de renovar ${ }^{17}$, influenció profundamente la actitud de la Iglesia, volviéndola a acercar al mundo del trabajo y de sus problemas según las claras intenciones reveladas por la encíclica papal Mater et Magistra del 15 de mayo de $1961^{18}$ - y determinando un nuevo enfoque sobre los problemas políticos italianos e internacionales, cuyos planteamientos serían precisados más tarde en una segunda encíclica: la Pacem in Terris del 11 de abril de 1963.

Mientras que durante el pontificado de Pío XII (1939-1958) las jerarquías eclesiásticas habían tomado parte activa en la vida pública italiana, influenciando la política de la DC y, a través de esa, la del País, el pontificado de Juan XXIII marcó un cambio claro de tendencias. En el bienio 1960-1961 muchos de los prelados que habían

${ }^{15}$ Cfr. MAMMARELLA, Giuseppe, op. cit., p. 257.

16 Ibídem.

${ }^{17}$ Cfr. DE MATTEI, Roberto, Il Concilio Vaticano II. Una storia mai scritta, Torino, Lindau, 2010, pp. 197-525.

${ }^{18}$ La encíclica, dirigida a «los venerables hermanos Patriarcas, Primados. Arzobispos, Obispos y otros Ordinarios en paz y comunión con la Sede apostólica», fue redactada en ocasión del $70^{\circ}$ aniversario de la Rerum Novarum de León XIII (promulgada el 15 de mayo de 1891) y pretendió analizar los «recientes progresos de la cuestión social, a la luz de la doctrina cristiana», dedicando una particular atención a las problemáticas de desarrollo económico. Cfr. Web oficial de la Santa Sede, URL:

<http://www.vatican.va/holy_father/john_xxiii/encyclicals/documents/hf_jxxiii_enc_150519 61_mater_it > [consultado el 10 de febrero 2013].

Como ha señalado Roberto de Mattei, los temas tratados en la encíclica hacen entender que uno de sus principales redactores fue monseñor Pietro Pavan, gran experto en doctrina social de la Iglesia y futuro cardenal. Cfr. DE MATTEI, Roberto, op. cit., p. 391. 
formado parte de lo que fue denominado "Pentágono Vaticano"19 fueron separados o perdieron la influencia ejercida hasta entonces. Esos cambios anunciaban que, con el nuevo pontífice, los intereses ecuménicos de la Iglesia prevalecerían sobre los particulares, y que la política seguida hasta entonces, de oposición hacia el mundo comunista, habría dejado espacio a nuevas y más abiertas tendencias, surgidas ante la necesidad de alcanzar un modus vivendi entre doctrinas opuestas. Esa nueva predisposición no pudo evitar reflejarse en la situación política italiana, por esto se tuvo la impresión de que los impedimentos que se habían expresado en el pasado con respecto al acercamiento entre fuerzas socialistas y católicas no se repetirían. Tal posibilidad otorgaba a la DC una mayor libertad en el desarrollo de su política y debilitaba a aquellos grupos conservadores que, en el seno del partido, estando vinculados al alto clero, habían sido los más fervientes portavoces de sus directivas.

Los motivos de la oposición de los comunistas al centro-izquierda son más complejos. El PCI no había ocultado nunca que aspiraba a ser el representante exclusivo de la clase trabajadora y sus relaciones con el PSI se habían siempre resentido por este telón de fondo competitivo, incluso en los momentos de más estrecha alianza. Con el diálogo entre socialistas y católicos el PCI no sólo perdía un importante aliado, sino que corría el riesgo de que le fueran arrebatadas posiciones y simpatías electorales. Entre la política comunista de «mesiánica espera de una conquista del poder que por el momento no tenía ninguna perspectiva» ${ }^{20}$ y la del PSI, que apuntaba a conseguir ventajas concretas y a crear nuevas posibilidades de progreso inmediato, la segunda habría podido imponerse y ganarse el favor de las clases trabajadoras, con el resultado de que se acentuase el aislamiento del PCI. Debido a las esperanzas y el interés que el diálogo entre PSI y DC suscitaba en vastos sectores del País, una actitud de abierta hostilidad hacia el centro-izquierda habría podido provocar desacuerdo y descontento también entre sus simpatizantes. Por ese motivo, los comunistas adoptaron una actitud de benévola espera que, sin embargo, estaba destinada a transformarse en una oposición gradual y cada vez más franca hacia el centro-izquierda, apenas éste se dispusiera a afrontar las primeras dificultades.

La actitud de la opinión moderada fue menos precisa en sus objetivos finales y en sus motivaciones; su oposición a la apertura a la izquierda parecía justificarse, por un

19 Fue en particular Carlo Falconi quien habló de "Pentágono Vaticano", refiriéndose a cinco importantes prelados (Nicola Canali, Giuseppe Pizzardo, Clemente Micara, Adeodato Piazza y Alfredo Ottaviani). Este grupo curial perseguía dos objetivos de fondo: la firme lucha contra la ideología comunista y la voluntad de realizar en Italia un amplio frente político moderado que pudiera contener la fuerza de los partidos de izquierda. Cfr. FALCONI, Carlo, Il Pentagono Vaticano, Bari, Laterza, 1958, pp. 31-70.

${ }^{20}$ MAMMARELLA, Giuseppe, op. cit., p. 259. 
lado, en la satisfacción por las condiciones presentes en el País y, por el otro, en la tradicional desconfianza del socialismo. Una economía en fase de expansión y niveles de bienestar crecientes, de los cuales se beneficiaban sobre todo las clases propietarias, explicaban las preferencias por un gobierno sustancialmente débil y con capacidad limitada de intervención. En realidad «la continuidad de un sistema del que era bien conocida la indulgencia en materia fiscal y en altas cuestiones administrativas [y] que permitiera el desarrollo de múltiples actividades a los márgenes de la ley, como la especulación inmobiliaria y la evasión fiscal, respondía a un claro interés de sectores medio-burgueses» ${ }^{21}$. En estos, como ya se ha dicho, estaba profundamente radicada la desconfianza hacia el socialismo. El hecho de que los socialistas hubieran abandonado sus tradicionales posiciones maximalistas y hubieran aceptado los principios de la democracia parlamentaria, se les presentaba como algo demasiado repentino para ser sincero: muchos pensaban que este nuevo posicionamiento ocultase una amenaza, y que el PSI hubiera asumido la función del "caballo de Troya", introduciendo al viejo aliado comunista al interno del gobierno.

La oposición al centro-izquierda parecía, por lo tanto, estaba motivada de forma diferente, de acuerdo a los intereses particulares de las clases sociales y de los grupos políticos. Esta doble oposición representará, primero en fase de preparación y luego, de ejecución, un gran obstáculo para los partidos del centro-izquierda, pero sobre todo para los socialistas, que se encontrarán entre los dos fuegos del recelo burgués y de la hostilidad, aunque encubierta, del PCI.

\section{Elecciones y disolución del Gobierno}

A principios de 1963, el cuarto gobierno de Fanfani había ejecutado todos los puntos principales de su programa. El 27 de noviembre de 1962, después de los últimos y obstinados tentativos de las derechas por bloquearla ${ }^{22}$, era aprobada la ley para la nacionalización de la industria eléctrica ${ }^{23}$, que preveía un ingente reembolso en cuotas

\footnotetext{
${ }^{21}$ MAMMARELLA, Giuseppe, op. cit., p. 259.

${ }^{22}$ Cfr. POMBENI, Paolo, op. cit., p. 235.

${ }^{23}$ La ley fue aprobada con una amplísima diferencia (404 votos a favor y apenas 74 en contra), gracias a la participación de todos los partidos de la mayoría parlamentaria y de los comunistas que, después de haber criticado algunos detalles, dieron su voto a favor. Cfr. D’AURIA, Elio, Gli anni della difficile alternativa. Storia della politica italiana 1956-1976, Napoli, Edizioni Scientifiche Italiane, p. 137.
} 
de diez años a las sociedades expropiadas ${ }^{24}$, y la constitución de un organismo de Estado: ENEL ${ }^{25}$. La medida, que representaba el mayor cumplimiento a las condiciones requeridas por los socialistas para la colaboración con el gobierno Fanfani, respondía a dos órdenes de necesidades: económicas y políticas. Desde el punto de vista económico, la nacionalización intentaba modificar una política de precios y de construcciones de nuevas instalaciones orientada al máximo beneficio $y$, por consiguiente, claramente desfavorable con las zonas económicamente deprimidas, a cuya revalorización había aspirado la política de todos los gobiernos en el último decenio. Desde el punto de vista político, la nacionalización tendía a redimensionar la capacidad de presión de los grandes monopolios privados, de los que las industrias eléctricas representaban la más típica expresión. Poco tiempo después, los socialistas admitirían que entre los dos objetivos, el que a ellos más les apremiaba era el segundo, dejándose entrever la fuerza de las tradicionales influencias ideológicas que aún pervivía en ellos ${ }^{26}$. La medida será objeto de fuertes críticas los años sucesivos, tanto por la manera en que fue ejecutada, como por las consecuencias psicológicas y económicas que acarreó, contribuyendo a llevar a gran parte de la opinión más cualificada de la izquierda democrática hacia nuevas vías de intervención y de control de la "mano pública" sobre la economía privada. Otra importante realización del primer gobierno de centro-izquierda, cuyo significado político no era menos relevante, fue el establecimiento de la Comisión Nacional para la programación económica, el 6 de agosto de $1962^{27}$. A ésta se le confiaba la labor de preparar un programa de desarrollo económico que tuviera como principales objetivos el eliminar el desequilibrio presente en los diferentes sectores productivos, entre las regiones desarrolladas y las subdesarrolladas y, asimismo, la desproporción de los consumos públicos y privados.

Con el espíritu de la nueva fórmula de centro-izquierda, se aprobaron otras dos medidas, a través de una ley de diciembre de 1962: un impuesto sobre los dividendos sobre acciones y otro sobre los perfiles de carácter inmobiliario. Ambas estaban dirigidas a actuar sobre la especulación, especialmente la que se había desarrollado en los últimos años, en las zonas edificables alrededor de las grandes ciudades en fase de rápida expansión, y que había aumentado artificiosamente los precios de los terrenos,

${ }^{24}$ El director de la Banca d'Italia Guido Carli fue el principal artífice de esta medida que fijó el importe de los resarcimientos en 1.500 mil millones de liras, a pagarse en tasas en el curso de diez años con un interés del 5,5\%. Cfr. VOULGARIS, Yannis, op. cit., p. 135.

25 Cfr. CRAVERI, Piero, La Repubblica dal 1958 al 1992, Torino, Utet, 1995, pp. 113-115.

${ }^{26}$ Cfr. MAMMARELLA, Giuseppe, op. cit., p. 279.

${ }^{27}$ La Comisión, presidida por el Ministro del Bilancio La Malfa, «estaba compuesta por nueve exponentes de las principales asociaciones sindicales (de los trabajadores y de la patronal), por nueve expertos de las mismas asociaciones y por trece expertos nombrados por el gobierno". TAMBURRANO, Giuseppe, op. cit., p. 166. 
haciendo imposible la construcción de casas populares a bajo precio ${ }^{28}$. Asimismo, la ley de pensiones, adquirió una particular importancia política ya que, a través de la aprobación de sustanciales aumentos para diferentes categorías de trabajadores, aumentaba el poder adquisitivo y, por tanto, la capacidad de consumo de las clases menos acaudaladas. En enero de 1963, se aprobó, además, la ley sobre la reorganización de la escuela, que incluía la instauración de una escuela media unificada, extendiendo la asistencia obligatoria hasta los 14 años de edad y limitando la enseñanza del latín, considerado una de las caracterizaciones clasistas del sistema educativo italiano.

El ordenamiento regional no se llevó a cabo porque en la DC predominó, en contra de la voluntad de Fanfani, la decisión de impedir su ejecución, ya que los socialistas no garantizaron explícitamente que no formarían gobiernos regionales con el apoyo del PCI ${ }^{29}$. A principios de 1963, la situación era de tal tensión que se tenía la impresión de estar cada vez más cerca de una crisis de gobierno. Esto se debía en parte al haber incumplido la creación de las regiones, pero también a las dificultades que muy a menudo surgían entre democristianos y socialistas, ya que los últimos querían alcanzar posiciones de poder en los organismos económicos y administrativos públicos que habían sido controlados por la DC durante numerosos años de gestión en el gobierno. PCI y MSI intentaron aprovechar estas dificultades presentando una moción de censura, que fue discutida entre del 24 al 26 de enero. Liberales y comunistas acusaron del "hundimiento", unos, a la DC y otros, al PSI, por su colaboración con el gobierno. Los partidos de la mayoría, después de haber confirmado la confianza, optaron por anticipar el final de la legislatura de mutuo acuerdo para tener así, cada uno, más posibilidades de atraer al respectivo electorado de referencia. Antes de la disolución de las Cámaras, establecida para el 18 de febrero, el Parlamento aprobó la ley constitucional que modificaba la composición y la duración del mandato de la Camera y del Senato, y que establecía para la legislatura del Senato la misma duración,

28 Elio d'Auria ha subrayado los efectos negativos de estas medidas que, en un breve periodo, acarrearon graves daños a la economía del País. En particular, las nuevas normas financieras en los dividendos sobre acciones, con la institución de la retención anticipada del 15\% (llamada cedolare d'acconto), provocaron una fuga de notables dimensiones de las inversiones en acciones que determinó una situación comatosa de la Bolsa, tanto que el gobierno se vio obligado, poco después de un año, a modificar la medida y a conceder la opción entre una retención del 30\%, cedolare secca, con el beneficio del anonimato (una vez que has cerrado y ajustado los dividendos) y una retención anticipada del $5 \%$, cedolare d'acconto, con la denuncia al fisco (sin haber ajustado los dividendos). Cfr. D'AURIA, Elio, op. cit., p. 137.

29 Algunos dirigentes democristianos temían la formación de regiones de mayoría comunista en el centro de Italia; otros, el coste excesivo de las nuevas estructuras administrativas; otros, incluso se referían a las autonomías regionales como un riesgo de disgregación del Estado, ante la unidad nacional. Cfr. MAMMARELLA, Giuseppe, op. cit., pp. 278-284. 
cinco años, que la de la Camera. Fue, además, aprobada la reducción del servicio militar a quince meses y se finalizaron las operaciones relativas a la nacionalización de la energía eléctrica, con el nombramiento del Consejo de Administración de ENEL, siendo designados presidente, Vito Antonio di Cagno y, vicepresidente, Luigi Grassini ${ }^{30}$.

La campaña electoral, considerada la más tranquila y fría que el País hubiese afrontado en la posguerra ${ }^{31}$, encontraba a los partidos del centro-izquierda en una posición difícil. A pesar de que el gobierno en su totalidad hubiera actuado con rapidez y decisión y los puntos fundamentales de su programa se hubieran realizado, «los problemas de fondo de la reorganización del Estado y de la economía aún no habían sido afrontados, ni habrían podido serlo, en el año que el gobierno había tenido a disposición, desde el momento de su nacimiento a la disolución de las Cámaras»32; a pesar el dinamismo que imprimió en el gobierno el presidente del Consiglio, Fanfani, el centro-izquierda no había conseguido ganarse la confianza del País y ampliar el respaldo popular. Los dos mayores partidos de la coalición, no solo no habían aclarado los objetivos inmediatos y a largo plazo de su programa de gobierno sino, al contrario, habían demostrado lo profundas que eran las divisiones y los antagonismos existentes entre ellos. Además, la apertura a la izquierda, había madurado mientras una profunda evolución económica y social estaba modificando las estructuras de la sociedad italiana, de modo que a los viejos problemas se añadían otros nuevos y la acción del gobierno chocaba inevitablemente con las fuerzas y los intereses que el proceso evolutivo estaba liberando33. Así, mientras por un lado, con su perspectiva de controles y de limitaciones, el centro-izquierda provocaba el descontento de los sectores privados y actuaba como freno para la expansión económica, por el otro, no había tenido tiempo ni energía suficiente para adoptar medidas que lograran influir en los profundos desequilibrios y las patentes injusticias que el crecimiento desordenado de la economía el País había producido.

Esa situación otorgó un terreno ideal para una campaña electoral de dos extremos. Las derechas aprovecharon el descontento de las clases medias, que se sentían amenazadas por el continuo aumento de los precios, consecuencia del boom económico y del creciente gasto público que había puesto en marcha una espiral de inflación. El PCI, después de una breve pausa ocasionada por el centro-izquierda,

\footnotetext{
$3^{3}$ Cfr. SCHIAVI, Giangiacomo, La rivoluzione elettrica. Enel, storia di una nazionalizzazione, Roma, Adnkronos, 1989, pp. 60-63.

${ }^{31}$ Cfr. MARTELLI, Evelina, L'altro atlantismo: Fanfani e la politica estera italiana (19581963), Milano, Guerrini e Associati, 2008, p. 441.

32 MAMMARELLA, Giuseppe, op. cit., p. 281.

33 Ibídem.
} 
volvió a la ofensiva intentando ganarse al electorado socialista, al que recriminaba la ruptura de la unidad de la clase obrera y la desigualdad de la política de centroizquierda. Gran parte del esfuerzo de persuasión del PCI se dirigió también hacia las masas agrícolas que, tras el proceso de industrialización, dejaron los campos para establecerse en las urbes de las zonas industriales del Norte, en condiciones de pobreza y tensión social. Una serie de escándalos de clientelismo político, que fueron hechos públicos a la vigilia de las elecciones ${ }^{34}$, favoreció la reacción de las oposiciones. Las derechas acusaron a la DC de desempeñar una política que estaba llevando a la desintegración del Estado y de las estructuras administrativas; los comunistas, por su parte, denunciaron la corrupción política que se nutría del monopolio del poder de la DC.

Los partidos de la mayoría, acorralados entre dos fuegos, no llegaron a combatir eficazmente esta doble ofensiva. El centro-izquierda, no tenía en su haber logros que pudieran hacerle ganar un amplio apoyo popular; por lo tanto, la estrategia del PSI, como la de la DC, fueron esencialmente defensivas y, más que ilustrar la importancia y las ventajas del centro-izquierda, intentaron desvincularse, admitiendo las carencias y mostrando una serie de ideas ambiguas y confusas.

Las elecciones, que tuvieron lugar el 28 y 29 de abril, se caracterizaron por una pérdida significativa de votos por parte de los actores principales del centro-izquierda. La DC, con el $38,3 \%$, perdió casi 750.000 votos respecto a las precedentes elecciones políticas de 1958. El PLI y el PCI con, respectivamente, el 7 y el 25,3\%, ganaron aproximadamente un millón de votos cada uno, mientras medio millón de votos más fueron a parar al Partido Socialdemocrático de Sagarat (6,1\%), que entre los de la mayoría había dirigido una hábil política de equilibrio entre la presión a la izquierda del PSI (13,8\%), y las resistencias de la Democracia Cristiana a la política reformista. El Partido Monárquico (3,1\%), registró una pérdida de aproximadamente 900.000 votos, que equivalía a uno verdadero derrumbe electoral; el MSI ganó 150.000 votos, mientras los republicanos permanecieron estacionarios con el 1,4\%35.

Para una más precisa valoración de los resultados es necesario tener presente el aumento del número de votantes en 1.200.000 personas, respecto a las elecciones de 1958. Valorando, por tanto, los resultados sobre la base de los porcentajes, se deduce que el mayor aumento había sido el del PLI, con el 3,5\% más respecto a 1958; seguía el

34 Cfr. GIOVAGNOLI, Agostino, Il partito italiano: la Democrazia Cristiana dal 1942 al 1994, Bari, Laterza, 1996, pp. 100-112.

35 Cfr. Archivio Storico del ministero dell'Interno, URL:

$<$ http://elezionistorico.interno.it/index.php?tpel $=$ C\&dtel=28/04/1963\&tpa $=$ I\&tpe $=A \& l e v O=0$

\&levsuto=o\&eso=S\&msS $>$ [consultado el 19 de febrero 2013]. 
PCI con el 2,6\%, y el PSDI con un 1,6\% más. La Democracia Cristiana, por su parte, sufrió las mayores pérdidas con una disminución del 4,1\%; seguían los monárquicos con el 3,2\%. Los otros partidos registraron variaciones mínimas: el MSI un aumento del $0,3 \%$, el PSI una bajada del $0,4 \%$ mientras los republicanos mantuvieron las posiciones $^{36}$.

Las reacciones del País al centro-izquierda provocaron una fuerte sacudida en el tradicional inmovilismo del electorado italiano, llegando a superar los pronósticos de la noche pre electoral, que preveían a grandes rasgos el desplazamiento de los votos, aún sin imaginar su real magnitud. Las razones de estos desplazamientos, precisamente por su consistencia, parecían bastante claras: la DC había perdido una notable porción del electorado moderado que, contrario al centro-izquierda, había pasado al PLI. La caída del Partido Monárquico se explicaba con las profundas transformaciones que se estaban produciendo en el Sur, donde el PNM había siempre tenido sus posiciones clientelares más fuertes; el electorado monárquico de base popular se dispersaba, pero la parte más considerable pasaba al PCI, al que iban también gran parte de los votos de los emigrantes meridionales en el Norte y, especialmente, en las mayores ciudades del triángulo industrial: Milán, Turín y Génova. Estos individuos, originarios de una condición socialmente atrasada y en reciente contacto con la realidad del mundo industrial y con un ambiente culturalmente ajeno, eran fácilmente atraídos en las múltiples organizaciones comunistas que les ofrecían asistencia material y una identidad política.

En general el voto de 1963, aún habiendo representado un hecho no completamente inesperado de las reacciones del electorado italiano al centro-izquierda, estaba lejos de constituir una prueba definitiva. El PSI, en líneas generales, había resistido bastante bien, compensando con ganancias respecto a la derecha, las pérdidas no sustanciales a la izquierda. La DC, tenía buenas posibilidades de reabsorber los votos que fueron a parar a la derecha, una vez que se hubieran amortiguado las reacciones más violentas al centro-izquierda. A los observadores más atentos no se les escapaba el hecho de que, a pesar de las gruesas ganancias en absoluto y en porcentaje, las derechas no podían constituir una posible alternativa a la Democracia Cristiana. Entre estos inteligentes observadores, estaba el mismo Amintore Fanfani. A pesar del evidente estancamiento que su política había recibido en las urnas, el estadista aretino interpretó el resultado electoral como una sustancial aprobación del centro-izquierda, por el hecho de que las profundas tendencias del País se habían modificado hacia la izquierda y la derecha ya no constituía, a su parecer, una alternativa posible de

${ }^{36}$ Cfr. TAMBURRANO, Giuseppe, op. cit., p. 164. 
gobierno37. Si esta interpretación contenía indudablemente una dosis de verdad, era también innegable que para la DC y para el presidente del Consiglio, se trataba de una dura derrota. El 16 de mayo Fanfani presentó su dimisión y la labor de resolver la crisis fue encomendada a Moro, que fracasó en el intento de presentar un centro-izquierda orgánico. Se constituyó un gobierno monocolor democristiano, presidido por Giovanni Leone. Más tarde, en el mes de diciembre, cuando fue posible dar vida a una coalición ministerial con la participación de los socialistas, a éste le sucedió el primer gobierno Moro, con el PSI finalmente integrado a pleno derecho en la mayoría de gobierno.

37 Cfr. MARTELLI, Evelina, L'altro atlantismo: Fanfani e la politica estera italiana (19581963), op. cit., p. 442.

El optimismo de Fanfani se deduce también de un balance sobre la actividad del gobierno que él redactó en marzo de 1963, un año después de la instauración de su cuarto Gabinete: «En conclusión: producción sostenida, aumento notable de la renta, disminución del desempleo, mejora de las retribuciones reales, son los resultados positivos que también en 1962 se han registrado, confirmando que el progreso económico continúa». FANFANI, Amintore, Centrosinistra '62, Milano, Garzanti, 1963, p. 130. 


\section{* El autor}

Matteo Anastasi ha cursado los estudios de Historia en la Universidad Europea de Roma presentando un trabajo de final de carrera sobre la actividad del cuarto gobierno de Amintore Fanfani, primer ministro italiano durante la crisis de los misiles de Cuba de 1962. En la actualidad realiza la especialización en Relaciones Internacionales en la Universidad LUISS Guido Carli, bajo tutela del profesor Francesco Perfetti. Se ha centrado en el análisis del fenómeno político de la apertura a la Izquierda en Italia tras la Segunda Guerra Mundial y en la historia diplomática italiana.

URL: < http://www.studistorici.com/progett/autori/\#Anastasi >

\section{Per citare questo articolo:}

ANASTASI, Matteo, «El Cuarto Gobierno de Fanfani y la apertura a la Izquierda. Un acercamiento histórico», Diacronie. Studi di Storia Contemporanea : Spazi, percorsi e memorie, 29/10/2013, URL:<http://www.studistorici.com/2013/10/29/anastasi_numero_15/ >

Diacronie Studi di Storia Contemporanea 8 www.diacronie.it

Risorsa digitale indipendente a carattere storiografico. Uscita trimestrale.

redazione.diacronie@hotmail.it

Comitato di redazione: Marco Abram - Jacopo Bassi - Luca Bufarale - Alessandro Cattunar - Elisa Grandi - Deborah Paci - Fausto Pietrancosta - Matteo Tomasoni - Luca Zuccolo 